Article

\title{
A Novel Tunable Multi-Frequency Hybrid Vibration Energy Harvester Using Piezoelectric and Electromagnetic Conversion Mechanisms
}

\author{
Zhenlong Xu ${ }^{1}$, Xiaobiao Shan ${ }^{1}$, Danpeng Chen ${ }^{2}$ and Tao Xie ${ }^{1, *}$ \\ Received: 31 October 2015; Accepted: 18 December 2015; Published: 5 January 2016 \\ Academic Editor: Sheng-Yuan Chu \\ 1 School of Mechatronics Engineering, Harbin Institute of Technology, Harbin 150001, China; \\ xuzhenlong2009@hotmail.com (Z.X.); shanxiaobiao@hit.edu.cn (X.S.) \\ 2 Zhejiang Guochen Robot Technology Co., Ltd., Hangzhou 311215, China; danpeng2@126.com \\ * Correspondence: xietao@hit.edu.cn; Tel.: +86-451-8641-7891; Fax: +86-451-8641-6119
}

\begin{abstract}
This paper presents a novel tunable multi-frequency hybrid energy harvester (HEH). It consists of a piezoelectric energy harvester $(\mathrm{PEH})$ and an electromagnetic energy harvester (EMEH), which are coupled with magnetic interaction. An electromechanical coupling model was developed and numerically simulated. The effects of magnetic force, mass ratio, stiffness ratio, and mechanical damping ratios on the output power were investigated. A prototype was fabricated and characterized by experiments. The measured first peak power increases by $16.7 \%$ and $833.3 \%$ compared with that of the multi-frequency EMEH and the multi-frequency PEH, respectively. It is 2.36 times more than the combined output power of the linear PEH and linear EMEH at $22.6 \mathrm{~Hz}$. The half-power bandwidth for the first peak power is also broadened. Numerical results agree well with the experimental data. It is indicated that magnetic interaction can tune the resonant frequencies. Both magnetic coupling configuration and hybrid conversion mechanism contribute to enhancing the output power and widening the operation bandwidth. The magnitude and direction of magnetic force have significant effects on the performance of the HEH. This proposed HEH is an effective approach to improve the generating performance of the micro-scale energy harvesting devices in low-frequency range.
\end{abstract}

Keywords: piezoelectric and electromagnetic; hybrid energy harvester; magnetic interaction; multi-frequency; low-frequency vibration

\section{Introduction}

Vibration energy harvesting is considered as a promising alternative approach to power wireless sensors nets and ultra low power microelectronics devices [1,2]. Piezoelectric and electromagnetic energy harvesting are the most reported transduction mechanisms. Traditional linear vibration energy harvester (VEH) has been usually considered as single degree-of-freedom (1DOF) model [3], which has a narrow operating frequency bandwidth. Once the excitation frequency shifts away from the resonant frequency, the performance of the VEH drops dramatically. In practice, the vibration source is random and time-varying. Some vibration sources especially exhibit multiple discrete frequency peaks in a large frequency range. For example, HVAC vents have three vibration frequencies of 21.8, 29 and $127.3 \mathrm{~Hz}$ [4]. Laptops exhibit two vibration frequencies of 43.2 and $90.2 \mathrm{~Hz}$ [5]. To effectively scavenge energy from vibration sources with multiple discrete frequencies is the first concerned research question. As we know, the resonant frequency of the piezoelectric energy harvester (PEH) increases sharply when the device size reduces to micro level. To date, most of the micro-scale PEHs operate at frequencies of more than $100 \mathrm{~Hz}$ [6,7], even at the level of $1 \mathrm{kHz}$ [8]. This limits their applications to harvest some ambient vibration energy, which occurs in the frequency range below 
$30 \mathrm{~Hz}$, such as those caused by wind or human motions. The micro electromagnetic energy harvester (EMEH) [9] exhibits lower power density due to the decreased flux density and smaller velocity of the magnet. Therefore, another concerning question is how to improve the performance of VEHs in the low frequency range.

To improve the performances of VEHs, a number of solutions have been proposed by researchers. These solutions include generator array [10-12], mechanical impact [13,14], nonlinear spring stiffness [15,16], magnetic coupling [17-20], hybrid conversion mechanism [21,22] and active/passive tuning techniques [23-25]. However, these generators are only effective in a continuous frequency range or at a single dominant resonant frequency. To harvest energy from vibration sources with discrete frequency peaks over a wide range, Chew and $\mathrm{Li}$ [26] reported a PEH composed by a series of piezoelectric beams, which are connected end-to-end. The resonant frequencies vary from 100 to $1000 \mathrm{~Hz}$ with different numbers of beams. Ferrari et al. [27] presented an array-type PEH made of three piezoelectric bimorphs. The respective three fundamental resonant frequencies are about 113, 183 and $281 \mathrm{~Hz}$. Yang et al. [28] designed a multi-frequency EMEH with three resonant modes of 369, 938 and $1184 \mathrm{~Hz}$. Yang et al. [29] added a nonlinear spring oscillator to a PEH to scavenge the multi-frequency vibration energy. Liu et al. [30] developed a multi-mode EMEH for three-dimensional (3D) excitation at different frequencies. Tadesse et al. [31] reported a hybrid energy harvester (HEH) using piezoelectric and electromagnetic mechanisms. It consisted of a piezoelectric cantilever beam and a permanent magnet attached at the tip, which oscillated within a stationary coil. The first and the second resonances of such device were $20 \mathrm{~Hz}$ and $300 \mathrm{~Hz}$. Wang et al. [32] and Shan et al. [33] analyzed the characteristics of the two degree-of-freedom (2DOF) HEH. Among these works, the resonant frequencies of VEHs are fixed when the prototypes are assembled. It is not convenient to tune the resonances according to the change of excitation frequency.

In this paper, we report a novel tunable multi-frequency $\mathrm{HEH}$, using piezoelectric and electromagnetic conversion mechanisms. The design procedure is explained by considering two generating elements coupled with magnetic interaction. An electromechanical coupling model of the $\mathrm{HEH}$ is established. The energy harvesting characteristics are numerically simulated and verified by experiments. The proposed HEH can effectively scavenge the vibration energy of two distributed resonant frequencies. Compared with the single energy harvesting mechanism, the proposed HEH not only generates more electric power but also broadens the operation frequency bandwidth in the low-frequency range. The magnetic interaction can be used to alter the resonant frequencies of HEH.

\section{Design and Working Principle}

Figure 1 illustrates the schematic diagram of the proposed tunable multi-frequency HEH. It consists of a cantilevered piezoelectric oscillator with a cylindrical magnet as a proof mass and a magnetic oscillator attached on the frame. Two oscillators are coupled through the magnetic interaction, which behaves as the repulsive force. An induction coil is attached around the magnetic oscillator. $R_{1}$ and $R_{2}$ are load resistances connected to piezoelectric patches and induction coil, respectively. When the HEH is subjected to the external excitation, the mechanical strain is converted into voltage in the piezoelectric layers. Meanwhile, the relative movement between the magnet and coil induces a current in the wire, based on Faraday's law. Note that the resonant frequencies of two oscillators are higher than their own natural frequencies due to the repulsive magnetic force. The natural frequency of the magnetic oscillator is designed to be lower than that of the piezoelectric oscillator, so that it can be used to scavenge the low-frequency vibration energy. Meanwhile, the piezoelectric oscillator can harvest the discrete high-frequency vibration energy.

The advantages of this design are as follows: (1) the output power is enhanced, because the kinetic energy can be simultaneously harnessed through piezoelectric effect and electromagnetic induction; (2) it is capable of simultaneously responding to excitation from multiple frequencies; (3) its resonant frequencies can be tuned by changing the separation distance between two magnets; (4) it has a wider bandwidth in the low-frequency range. 


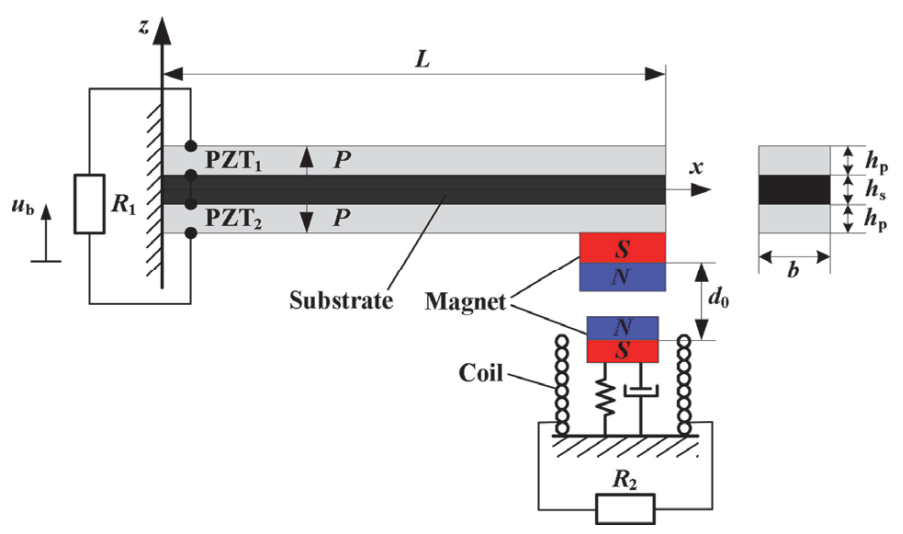

Figure 1. Schematic diagram of the proposed hybrid energy harvester.

\section{Theoretical Modeling}

\subsection{Electromagnetic Coupling Coefficient and Magnetic Force}

The electromagnetic transduction mechanism is based on Faraday's law of induction. In this paper, the magnetic oscillator is arranged to move inside the induction coil along the center axis, as depicted in Figure 2. The electromotive force $U_{\mathrm{emf}}$ is derived from the rate of change of the magnetic flux $\Phi_{B}$ as follows [34]

$$
U_{\mathrm{emf}}=-\frac{\mathrm{d} \Phi_{B}}{\mathrm{~d} t}=-\frac{\mathrm{d} \Phi_{B}}{\mathrm{~d} z} \cdot \frac{\mathrm{d} z}{\mathrm{dt}}=-\frac{A \mathrm{~d} B}{\mathrm{~d} z} \cdot \dot{z}=\theta_{\mathrm{em}} \dot{z}
$$

where $B$ is the magnetic flux density. $A$ is the area enclosed by the wire loop, which is considered as a constant value. $\theta_{\mathrm{em}}$ is called the electromagnetic coupling coefficient.

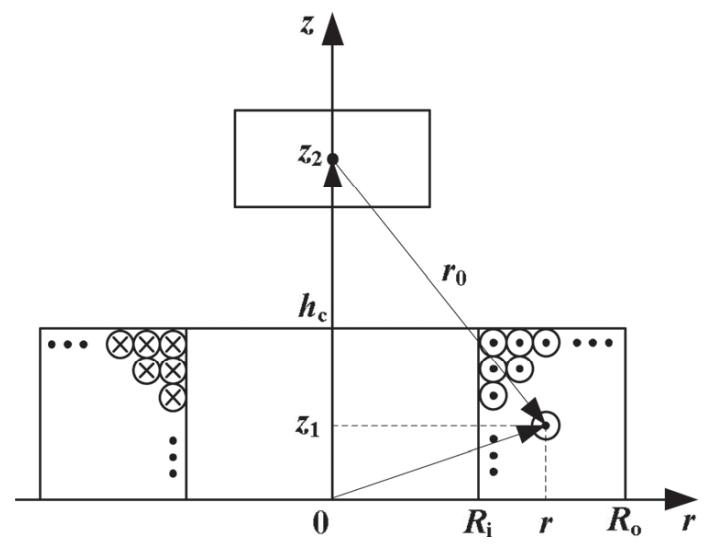

Figure 2. The relative position between magnet and coil.

The magnet is considered as a dipole with magnetic moment vector $m_{2}$ [35], the magnetic field generated by the magnet can be expressed as

$$
\boldsymbol{B}=-\frac{\mu_{0}}{4 \pi}\left[\frac{m_{2}}{r_{0}^{3}}-\frac{3 r_{0}\left(\boldsymbol{m}_{2} \cdot r_{0}\right)}{r_{0}^{5}}\right]
$$

where $\mu_{0}=4 \pi \times 10^{-7} \mathrm{Hm}^{-1}$ is the permeability of vacuum. $r_{0}$ is the distance vector from the center of the magnet to an arbitrary single wire. Assumed that the position coordinates of an arbitrary wire and magnet core are $\left(r, z_{1}\right)$ and $\left(0, z_{2}\right)$, respectively. The corresponding expression of $\boldsymbol{B}$ is 


$$
\boldsymbol{B}=\frac{B_{r} V_{2}}{4 \pi} \cdot \frac{3\left(z_{1}-z_{2}\right) r \boldsymbol{e}_{r}-\left[r^{2}-2\left(z_{1}-z_{2}\right)^{2}\right] \boldsymbol{e}_{z}}{\left[r^{2}+\left(z_{1}-z_{2}\right)^{2}\right]^{5 / 2}}
$$

where $B_{r}$ and $V_{2}$ are the residual magnetic flux density and volume of the source magnet. $\boldsymbol{e}_{r}$ and $\boldsymbol{e}_{z}$ are the unit vectors along $r$ - and $z$-axis, respectively.

As can be seen from Equation (1), the magnetic flux gradient along the $z$-axis direction is related to the induced voltage. For this reason, the magnetic flux density along the $z$-axis is the focus of research. The magnetic flux through the area enclosed by the wire is

$$
\Phi=\oint_{s} \boldsymbol{B} \mathrm{d} \boldsymbol{A}=\frac{B_{r} V_{2} r^{2}}{2\left[r^{2}+\left(z_{1}-z_{2}\right)^{2}\right]^{3 / 2}}
$$

The average magnetic flux over the cross-section of the coil can be given as

$$
\bar{\Phi}=\frac{1}{A_{c}} \cdot \int_{0}^{h_{c}} \int_{R_{\mathrm{i}}}^{R_{\mathrm{o}}} \Phi \mathrm{d} r \mathrm{~d} z_{1}
$$

where $A_{\mathrm{c}}=\left(R_{\mathrm{o}}-R_{\mathrm{i}}\right) h_{\mathrm{c}}$ is the coil cross-sectional area [36]. $h_{\mathrm{c}}, R_{\mathrm{o}}$, and $R_{\mathrm{i}}$ are the height, outer radius, and inner radius of the coil, respectively. Correspondingly, the total magnetic flux through the coil can be derived from $\Phi_{B}=f_{\mathrm{c}} N \bar{\Phi} . f_{\mathrm{c}}=N A_{\mathrm{w}} / A_{\mathrm{c}}$ is the coil fill factor [37]. $N$ is the number of turns in the coil. $A_{\mathrm{W}}$ is the cross-sectional area of single wire. Consequently, the electromotive force $\mathrm{U}_{\mathrm{emf}}$ can be expressed as

$$
U_{\mathrm{emf}}=-\frac{\mathrm{d}\left(f_{\mathrm{c}} N \bar{\Phi}\right)}{\mathrm{d} z} \dot{z}=\theta_{\mathrm{em}} \dot{z}
$$

where $\theta_{\mathrm{em}}$ is

$$
\begin{gathered}
\theta_{\mathrm{em}}=-\frac{B_{r} V_{2} f_{\mathrm{c}} N}{2 A_{\mathrm{c}}}\left[\ln \frac{R_{\mathrm{i}}+\sqrt{R_{\mathrm{i}}^{2}+\left(z_{2}-h_{\mathrm{c}}\right)^{2}}}{R_{\mathrm{o}}+\sqrt{R_{\mathrm{o}}^{2}+\left(z_{2}-h_{\mathrm{c}}\right)^{2}}+\ln \frac{R_{\mathrm{o}}+\sqrt{R_{\mathrm{o}}^{2}+z_{2}^{2}}}{R_{\mathrm{i}}+\sqrt{R_{\mathrm{i}}^{2}+z_{2}^{2}}}+}\right. \\
\left.\frac{R_{\mathrm{o}}}{\sqrt{R_{\mathrm{o}}^{2}+\left(z_{2}-h_{\mathrm{c}}\right)^{2}}}-\frac{R_{\mathrm{o}}}{\sqrt{R_{\mathrm{o}}^{2}+z_{2}^{2}}}-\frac{R_{\mathrm{i}}}{\sqrt{R_{\mathrm{i}}^{2}+\left(z_{2}-h_{\mathrm{c}}\right)^{2}}}+\frac{R_{\mathrm{i}}}{\sqrt{R_{\mathrm{i}}^{2}+z_{2}^{2}}}\right]
\end{gathered}
$$

It is assumed that the centers of the two magnets are always vertically aligned during the vibration of the HEH. The magnetic force between the magnetic proof mass and magnetic oscillator can be calculated based on the dipole-dipole model [35], which is given as

$$
F_{\mathrm{m}}=\frac{3 \mu_{0} m_{1} m_{2}}{2 \pi\left[u_{1}-u_{2}+d_{0}\right]^{4}}
$$

where $m_{1}$ is the magnitude of the magnetic dipole moment of magnetic proof mass. $u_{1}$ and $u_{2}$ are the displacements of the magnetic proof mass and magnetic oscillator relative to the base, respectively. $d_{0}$ is the initial separation distance between the magnetic dipoles. The direction of the magnetic force depends on the magnetization directions of the magnets.

Under the small-amplitude excitation, the difference of the displacements $\left(u_{1}-u_{2}\right)$ is small. The Taylor's expansion of magnetic force $F_{\mathrm{m}}$ around $\left(u_{1}-u_{2}\right)$ can be expressed as

$$
F_{\mathrm{m}}=\frac{3 \mu_{0} m_{1} m_{2}}{2 \pi}\left[d_{0}^{-4}+(-4) d_{0}^{-5}\left(u_{1}-u_{2}\right)+10 d_{0}^{-6}\left(u_{1}-u_{2}\right)^{2}+\cdots+\frac{F_{\mathrm{m}}^{(n)}(0)}{n !}\left(u_{1}-u_{2}\right)^{n}\right]
$$


If the high-order terms $(n \geqslant 2)$ are neglected, the magnetic force will only induce the linear equivalent stiffness to the harvester, just as a linear spring.

\subsection{Modeling of Linear $1 D O F$ PEH}

When both induction coil and magnetic oscillator are removed, the HEH becomes a conventional 1DOF linear PEH (LPEH). The LPEH consists of a bimorph piezoelectric cantilever beam and a proof mass. The electromechanical coupling model can be derived from the energy method [38,39], which is based on the modal analysis and small-signal piezoelectric material constitutive law. The proof mass is modeled as a mass point and rotation inertia is neglected. The beam is considered to be an Euler-Bernoulli beam, whose shear deformation and rotation inertia are neglected. The piezoelectric patches are assumed to be perfectly bonded with the substrate and the thicknesses of the electrodes are neglected. The electrical field is assumed to be constant across the thickness of the piezoelectric element. Geometric nonlinearity of the beam is ignored under the assumption of small deflection. Considering that the frequency of excitation source in the environment is below $200 \mathrm{~Hz}$ [40], the fundamental mode of the PEH is the research focus.

Assume that the displacement of the base excitation is $u_{\mathrm{b}}(t)$. In the Rayleigh-Ritz approach, the displacement of the PEH relative to the base $u(x, t)$ and electrical potential for the electrode pair $\varphi(z, t)$ can be written as

$$
\begin{aligned}
& u(x, t)=\psi_{r}(x) r(t) \\
& \varphi(z, t)=\psi_{v}(z) v(t)
\end{aligned}
$$

where $x$ is the axial position coordinate of the beam. $\psi_{r}(x)$ is the mechanical mode shape function. $r(t)$ is the modal coordinate. $\psi_{v}(z)$ is the electrical potential distribution function. $v(t)$ is the electrical temporal coordinate.

The electromechanical coupling governing equations are simplified as

$$
\left\{\begin{array}{c}
M \ddot{r}+C \dot{r}+K r-\theta_{\mathrm{p}} v_{1}=-B_{\mathrm{f}} \ddot{u}_{\mathrm{b}} \\
\theta_{\mathrm{p}} \dot{r}+C_{p} \dot{v}_{1}+v_{1} / R_{1}=0
\end{array}\right.
$$

where the mass $M$, stiffness $K$, piezoelectric coupling coefficient $\theta_{\mathrm{p}}$, capacitive $C_{\mathrm{p}}$, and forcing factor $B_{\mathrm{f}}$ are obtained as follows

$$
\begin{gathered}
M=\int_{0}^{L} m \psi_{r}^{2} \mathrm{~d} x+M_{t} \psi_{r}^{2}(L) \\
K=\int_{V_{\mathrm{s}}} c_{\mathrm{s}}\left(-z \psi_{r}^{\prime \prime}\right)^{2} \mathrm{~d} V_{\mathrm{s}}+\int_{V_{\mathrm{p}}} c_{11}^{\mathrm{E}}\left(-z \psi_{r}^{\prime \prime}\right)^{2} \mathrm{~d} V_{\mathrm{p}} \\
\theta_{\mathrm{p}}=\int_{V_{\mathrm{p}}} e_{31}\left(-z \psi_{r}^{\prime \prime}\right)\left(-\nabla \psi_{v}\right) \mathrm{d} V_{\mathrm{p}} \\
C_{\mathrm{p}}=\int_{V_{\mathrm{p}}} \varepsilon_{33}^{\mathrm{S}}\left(-\nabla \psi_{v}\right)^{2} \mathrm{~d} V_{\mathrm{p}} \\
B_{\mathrm{f}}=\int_{0}^{L} m \psi_{r} \mathrm{~d} x+M_{t} \psi_{r}(L)
\end{gathered}
$$

where the subscripts $\mathrm{p}$ and $\mathrm{s}$ indicate the piezoelectric element and substrate, respectively. $V_{\mathrm{p}}$ and $V_{\mathrm{s}}$ denote the volumes. $M_{t}$ is the proof mass. $m$ is the mass per length in the axial direction. $z$ indicates the distance from the neutral axis of the piezoelectric beam. $v_{1}$ is the voltage across external resistance $R_{1}$ for the piezoelectric patches. $\ddot{u}_{\mathrm{b}}$ is the excitation acceleration. $C$ denotes the mechanical damping, which can be measured by the logarithmic decrement method or bandwidth method [41]. The subscript 1 corresponds to the $x$ direction and 3 corresponds to the $z$ direction. $c, e$, and $\varepsilon$ are the modulus of elasticity, piezoelectric constant, and dielectric constant, respectively. $\nabla$ is the differential operator. 


\subsection{Modeling of the Proposed HEH}

The electromagnetic energy harvesting part can be simplified as a 1DOF spring-mass-damper system, as shown in Figure 1. The magnetic force is considered as a concentrated force applied on the centers of both magnets along the $z$-axis direction. Assume that a distributed force $F(x, t)$ is applied on the beam. Based on the modal analysis, the corresponding modal force for the fundamental vibration mode, $F_{1}$, can be expressed as

$$
F_{1}=\int_{0}^{L} F(x, t) \psi_{r}(x) \mathrm{d} x
$$

Accordingly, the modal magnetic force applied on the piezoelectric beam is given as

$$
F_{1}=F_{\mathrm{m}}(L-R, t) \psi_{r}(L-R)
$$

where $R$ is the radius of the magnetic proof mass.

Due to the low excitation frequency, the coil inductance is ignored [42]. Combing Equations (12) and (19), the electromechanically coupling governing equations of the $\mathrm{HEH}$ for the fundamental mode can be derived as

$$
\left\{\begin{array}{c}
M \ddot{r}+C \dot{r}+K r-\theta_{\mathrm{p}} v_{1}-F_{\mathrm{m}} \psi_{r}(L-R)=-B_{\mathrm{f}} \ddot{u}_{\mathrm{b}} \\
M_{2} \ddot{u}_{2}+C_{2} \dot{u}_{2}+K_{2} u_{2}+\theta_{\mathrm{em}} I_{2}+F_{\mathrm{m}}=-M_{2} \ddot{u}_{\mathrm{b}} \\
\theta_{\mathrm{p}} \dot{r}+C_{\mathrm{p}} \dot{v}_{1}+v_{1} / R_{1}=0 \\
\theta_{\mathrm{em}} \dot{u}_{2}-\left(R_{\mathrm{c}}+R_{2}\right) I_{2}=0
\end{array}\right.
$$

where $M_{2}, C_{2}$, and $K_{2}$ are the lumped mass, mechanical damping, and spring stiffness of the magnetic oscillator, respectively. $R_{\mathrm{c}}$ is the internal resistance of the coil.

Letting

$$
\omega_{1}=\sqrt{K / M}, \quad \omega_{2}=\sqrt{K_{2} / M_{2}}, \quad \zeta_{1}=\frac{C}{2 \sqrt{K M}}, \quad \zeta_{2}=\frac{C_{2}}{2 \sqrt{K_{2} M_{2}}}
$$

and defining a state vector $\mathbf{X}=\left[\begin{array}{lllll}X_{1} & X_{2} & X_{3} & X_{4} & X_{5}\end{array}\right]^{t}=\left[\begin{array}{lllll}r & \dot{r} & u_{2} & \dot{u}_{2} & v_{1}\end{array}\right]^{t}(t$ denotes the transpose of the vector), the governing Equation (20) can be written in the state space form as

$$
\dot{\mathbf{X}}=\left[\begin{array}{c}
\dot{r} \\
\ddot{r} \\
\dot{u}_{2} \\
\ddot{u}_{2} \\
\dot{v}_{1}
\end{array}\right]=\left[\begin{array}{c}
X_{2} \\
-\omega_{1}^{2} X_{1}-2 \omega_{1} \zeta_{1} X_{2}+\theta_{\mathrm{p}} X_{5} / M+F_{\mathrm{m}} \psi_{r}(L-R) / M-B_{\mathrm{f}} \ddot{u}_{\mathrm{b}} / M \\
X_{4} \\
-\omega_{2}^{2} X_{3}-\left(2 \omega_{2} \zeta_{2}+C_{\mathrm{em}} / M_{2}\right) X_{4}-F_{\mathrm{m}} / M_{2}-\ddot{u}_{\mathrm{b}} \\
-\theta_{\mathrm{p}} X_{2} / C_{\mathrm{p}}-X_{5} /\left(R_{1} C_{\mathrm{p}}\right)
\end{array}\right]
$$

where $C_{\mathrm{em}}=\theta_{\mathrm{em}}^{2} /\left(R_{2}+R_{\mathrm{c}}\right)$ is the electromagnetic damping.

The output power delivered to the external loads $R_{1}$ and $R_{2}$ are respectively given as

$$
\begin{gathered}
P_{\mathrm{p}}=\frac{1}{T} \int_{0}^{T} \frac{v_{1}^{2}}{R_{1}} \mathrm{~d} t \\
P_{\mathrm{em}}=\frac{1}{T} \int_{0}^{T}\left(\frac{\theta_{\mathrm{em}} \dot{\mathcal{u}}_{2}}{R_{2}+R_{\mathrm{c}}}\right)^{2} R_{2} \mathrm{~d} t
\end{gathered}
$$

where $T=2 \pi / \omega$ is the cycle of the base excitation. $\omega$ is the angular velocity. The total output power of the $\mathrm{HEH}$ is

$$
P=P_{\mathrm{p}}+P_{\mathrm{em}}
$$

\section{Numerical Simulation}

Upon Equations (22)-(24), the performances of the HEH depend on the magnetic force, mass, stiffness, and mechanical dampings of the system. In this section, we will discuss the effects of the 
above parameters on the harvesting performances of the HEH. The theoretical model is numerically simulated by using the ordinary differential equation solver "ode 45 " in MATLAB ${ }^{\circledR}$ (MathWorks, Natick, MA, USA). The piezoelectric material used is PZT-5H [43]. The geometric and physical properties of the proposed HEH are given in Table 1. Note that magnet A stands for the proof magnet, while magnet $B$ stands for the magnetic oscillator. In the following parts, the electromechanical coupling coefficients $\theta_{\mathrm{p}}$ and $\theta_{\mathrm{em}}$ are determined based on these properties. The reference resistances connected to the HEH are assumed to be $R_{1}=1 / C_{\mathrm{p}} \omega_{1}, R_{2}=R \mathrm{c}=240 \Omega$. The excitation acceleration is set $2 \mathrm{~m} / \mathrm{s}^{2}$ and the initial separation distance $d_{0}$ is $52.5 \mathrm{~mm}$. The equilibrium position of the center of magnetic oscillator is set on the upper surface of the coil, i.e., $z_{2}=h_{\mathrm{c}}$. The mechanical damping ratio of each oscillator is set 0.02 .

Table 1. Geometric and physical properties of the hybrid energy harvester (HEH).

\begin{tabular}{lc}
\hline \multicolumn{1}{c}{ Parameter } & Value \\
\hline PZT length $\times$ width $\times$ thickness $\left(\mathrm{mm}^{3}\right)$ & $50 \times 20 \times 0.2$ \\
PZT density $\left(\mathrm{kg} / \mathrm{m}^{3}\right)$ & 7500 \\
Young's modulus of PZT layer $(\mathrm{GPa})$ & 60.6 \\
Substrate length $\times$ width $\times$ thickness $\left(\mathrm{mm}^{3}\right)$ & $50 \times 20 \times 0.24$ \\
Substrate density $\left(\mathrm{kg} / \mathrm{m}^{3}\right)$ & 8920 \\
Young's modulus of substrate $(\mathrm{GPa})$ & 90 \\
Magnet A radius $\times$ thickness $\left(\mathrm{mm}^{2}\right)$ & $10 \times 10$ \\
Magnet B outer radius $\times$ inner radius $\times$ thickness $\left(\mathrm{mm}^{3}\right)$ & $9 \times 4 \times 7$ \\
Magnet density $\left(\mathrm{kg} / \mathrm{m}^{3}\right)$ & 7800 \\
Residual magnetic flux density $(\mathrm{T})$ & 1.25 \\
Spring stiffness $(\mathrm{N} / \mathrm{m})$ & 220 \\
\hline
\end{tabular}

\subsection{Electromagnetic Coupling Coefficient}

Figure 3 shows the relationship between electromagnetic coupling coefficient $\theta_{\mathrm{em}}$ and position of magnetic oscillator $z_{2}$. It seems that $\theta_{\mathrm{em}}$ varies nonlinearly with the change of $z_{2}$ due to the change of magnetic field. $\theta_{\mathrm{em}}$ reaches the peak values around the upper and lower surfaces of the coil, while the value is zero at the center of the coil. Because the maximum velocity of the magnetic oscillator occurs at the static balance position during the harmonic oscillation, the upper and lower surfaces of the coil are optimal equilibrium positions for the magnetic oscillator.

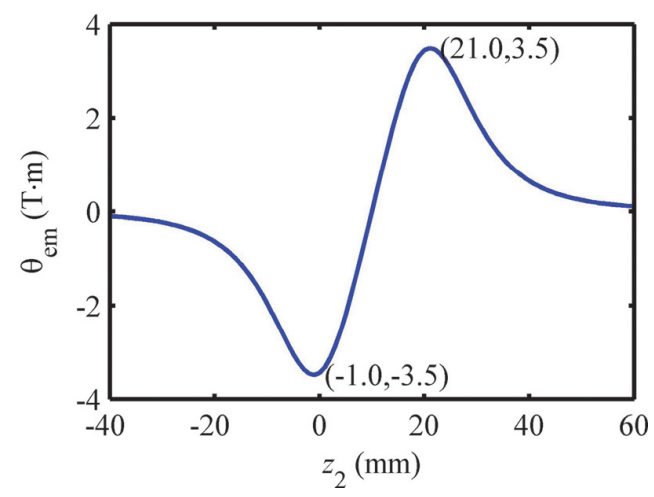

Figure 3. Electromagnetic coupling coefficient versus $z_{2}$.

\subsection{Effect of Magnetic Force on the Output Power}

Figure 4 shows the effect of magnetic force on the output power of the HEH. The first and second resonant frequencies are induced by magnetic and piezoelectric oscillators, respectively. It is the typical characteristic of the multi-frequency VEH. It can be seen that both of the two resonant frequencies increase with the decreasing of $d_{0}$, which is the same as the tendency of the difference 
of the two resonant frequencies. Therefore, we can tune the resonant frequencies of the system by changing the initial separation distance $d_{0}$. However, when $d_{0}$ is small enough and the repulsive force is large enough, the two resonances will merge into one resonance. In this situation, the magnetic interaction acts as a rigid connection between two oscillators, so that two oscillators have the same resonant frequency around the $\omega_{1}$. With the decrease of $d_{0}$, the magnitude of the first peak power gradually increases, while the one of the second peak constantly decreases. When the second peak finally disappears, the first peak reaches the maximum value. Although the magnetic force is large enough when $d_{0}=10.0 \mathrm{~mm}$, there is still no obvious hardening or softening response, which is the typical phenomenon in magnetic coupling nonlinear VEH [20]. The main reason is that the difference of displacements between two oscillators $\left(u_{1}-u_{2}\right)$ is very small under the small-amplitude excitation, so that the magnetic nonlinearity is invisible, which can be referred in Equation (9).

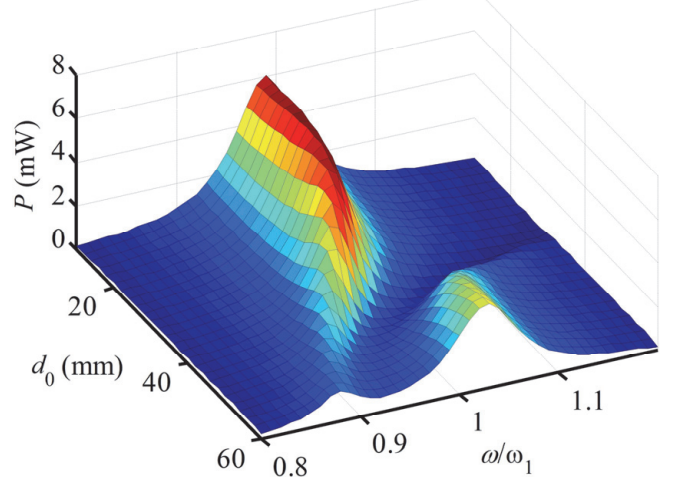

Figure 4. Output power versus excitation frequency for different $d_{0}$.

\subsection{Effect of Mass Ratio on the Output Power}

Define $M_{1}=M / \psi_{r}^{2}(L), K_{1}=K / \psi_{r}^{2}(L)$ [17], $\mu=M_{2} / M_{1}$, and $\alpha=K_{2} / K_{1}$, where $M_{1}$ and $K_{1}$ are the lumped mass and stiffness of the piezoelectric oscillator, respectively. $\mu$ and $\alpha$ are mass and stiffness ratios, respectively. According to the properties in Table $1, M_{1}=0.0257 \mathrm{~kg}, K_{1}=651.8 \mathrm{~N} / \mathrm{m}, M_{2}=0.0118$ $\mathrm{kg}, K_{2}=220.0 \mathrm{~N} / \mathrm{m}, \mu=0.46$, and $\alpha=0.34$. The output power of the HEH versus excitation frequency for different mass ratio $\mu$ is illustrated in Figure 5. For a given $\alpha$, the first resonance shifts to a higher frequency with the decrease of $\mu$. It is difficult to distinguish the tendency of the second resonant frequency, due to the mixture of two peak power. Because the HEH is used in the low-frequency environment, the dynamic characteristic of the first peak power is our research focus. The magnitude of first peak initially decreases and then increases as $\mu$ decreases. Finally, it reduces to a smaller value when the first resonance is higher enough than the second one. In a word, the mass ratio affects the resonance distribution and output power of the $\mathrm{HEH}$.
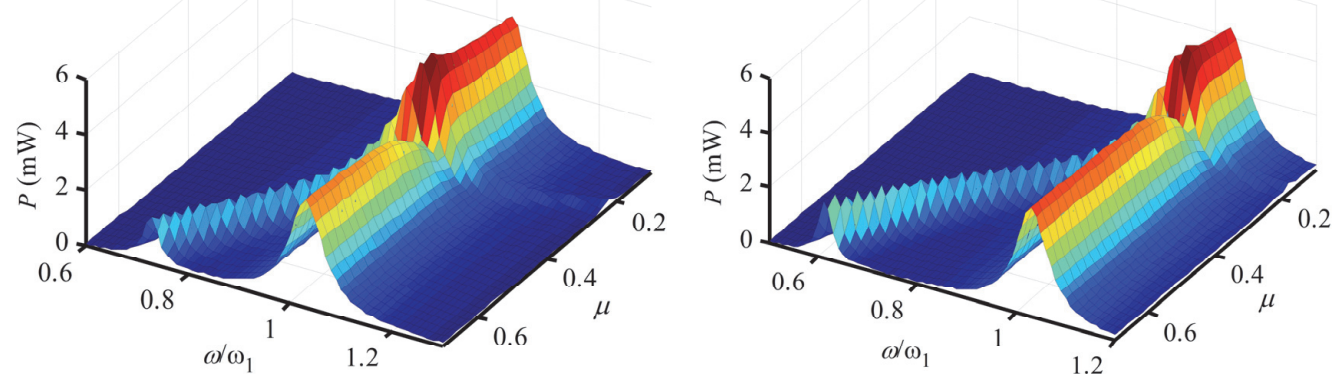

Figure 5. Output power versus excitation frequency for different $\mu$ : (a) $\alpha=0.34$; (b) $\alpha=0.23$. 
For the given $\mu$, the magnitude of the first peak power is enhanced with the smaller $\alpha(\alpha=0.23)$, which is contrary to the change of the second peak power. However, the first resonance shifts to a lower value with the decreasing of $\alpha$. Under the same excitation condition, the smaller $\alpha$ is, the more vibration energy can be harvested by magnetic oscillator. The second resonant frequency remains almost unchanged.

\subsection{Effect of Stiffness Ratio on the Output Power}

Figure 6 depicts the effect of stiffness ratio $\alpha$ on the output power of the HEH. For a given $\mu$, the first resonant frequency increases as $\alpha$ increases, while the magnitude of the first peak initially decreases, then increases, and finally reduces to a smaller value. It also shows that $\alpha$ has almost no effect on the second resonance. When $\alpha$ remains unchanged, the larger $\mu$ is, the more the magnitude of the first peak increases. This conclusion is identical with that obtained before. Therefore, the stiffness ratio can also be used to alter the performance of the $\mathrm{HEH}$.
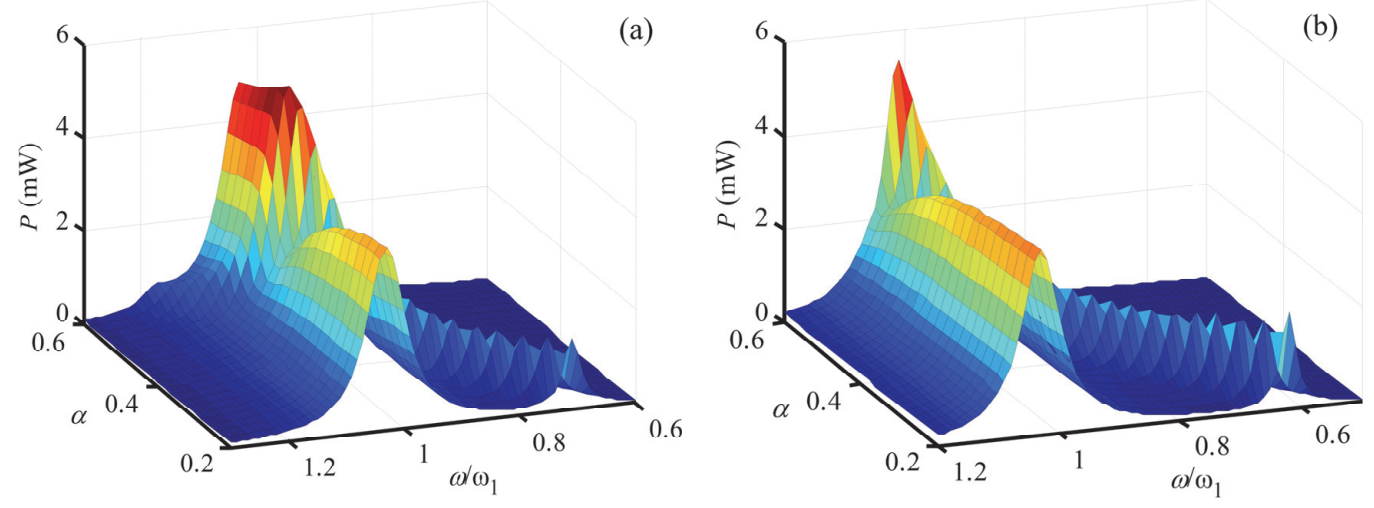

Figure 6. Output power versus excitation frequency for different $\alpha$ : (a) $\mu=0.46$; (b) $\mu=0.60$.

\subsection{Effects of Mechanical Damping Ratios on the Output Power}

Figure 7 shows the effects of mechanical damping ratios $\zeta_{1}$ and $\zeta_{2}$ on the output power of the $\mathrm{HEH}$, respectively. It can be seen that $\zeta_{1}$ mainly affects the performance of the second peak induced by the piezoelectric oscillator, while $\zeta_{2}$ mainly affects that of the first peak induced by the magnetic oscillator. With the increase of $\zeta_{1}$, the magnitude of the second peak output power constantly decreases, but the bandwidth increases. The magnitude of the first peak power slightly decreases. These effects are the same as that induced by $\zeta_{2}$.
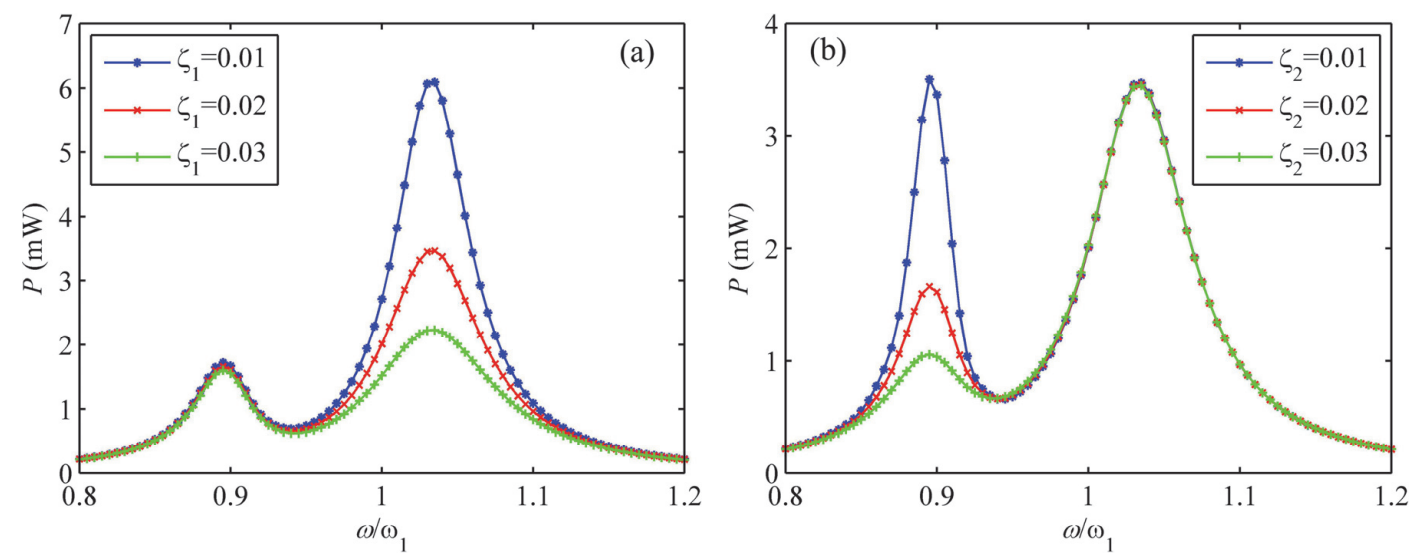

Figure 7. Output power versus excitation frequency for different mechanical damping ratios: (a) $\zeta_{1} ;$ (b) $\zeta_{2}$. 


\section{Experimental Method}

Figure 8 shows the fabricated macro-scale prototype of the proposed HEH and the experimental system. The substrate of the cantilever beam is made of Phosphor Bronze (ALB Copper Alloys Co., Ltd., Xiamen, China). It is sandwiched between two PZT-5H patches (Baoding Hongsheng Acoustics Electron Apparatus Co., Ltd., Baoding, China), which are connected in series. Two permanent magnets are NdFeB (Ningbo Hony Technology Co.,Ltd., Ningbo, China). They have opposite directions of magnetization. The magnetic proof mass is cylindrical. The magnet of the magnetic oscillator is a magnetic ring. It is attached to an aluminum rod, which is bolted on the frame. The rod is used to keep the magnet moving on the vertical direction. The copper wire coil (Changzhou Wujin Enameled Wire Factory Co., Ltd., Changzhou, China) is placed along the motion direction of the magnetic oscillator. The geometric and physical properties of the prototype are according to Table 1 . The internal resistance of the coil is $240 \Omega$. The experimental system consists of an electromagnetic shaker (JZK-50) (Sinocera Piezotronics Inc., Yangzhou, China), a signal generator (DG-1022) (Rigol Technologies Inc., Beijing, China), a power amplifier (YE5874A) (Sinocera Piezotronics Inc., Yangzhou, China). The base excitation acceleration was measured by an accelerometer (YD64-310) (Qinhuangdao Xinheng Electronic Technology Co. Ltd., Qinhuangdao, China). The output voltage was continuously monitored by the oscilloscopes DS-5102MA (Rigol Technologies Inc., Beijing, China) and TDS-1012C (Tektronix China Ltd., Shanghai, China).

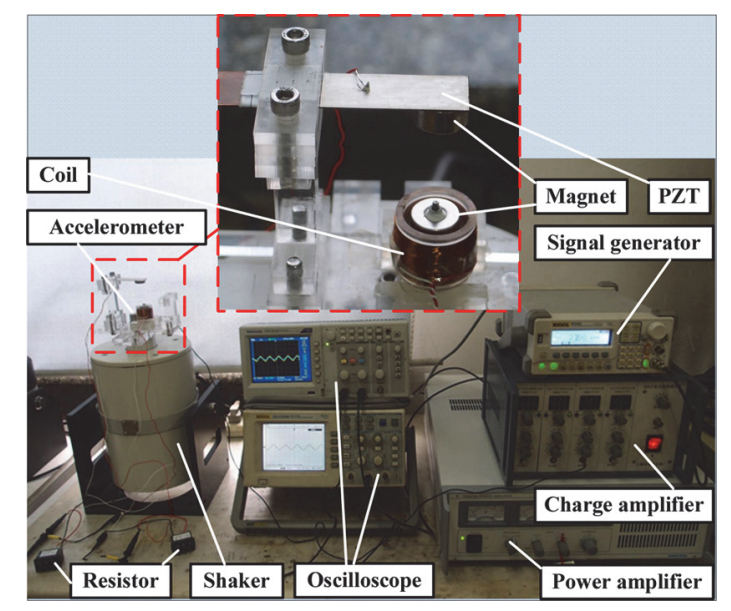

Figure 8. Prototype of the HEH and the experimental system.

Upon the numerical simulation, the theoretical resonances of the piezoelectric and magnetic oscillators are about $26 \mathrm{~Hz}$ and $22 \mathrm{~Hz}$, respectively. Thus, we sweep the excitation frequency from 16 to $32 \mathrm{~Hz}$ in the following experiments. The base excitation generated by the electromagnetic shaker is harmonic and the acceleration is set $2 \mathrm{~m} / \mathrm{s}^{2}$. The initial separation distance between two magnets $d_{0}$ is $52.5 \mathrm{~mm}$.

In order to evaluate the performance of the proposed $\mathrm{HEH}$, four different energy harvesters with single conversion mechanism were also developed and experimentally verified for comparison under the same excitation condition. When the induction coil around the magnetic oscillator is open-circuit, there will be no electromagnetic damping induced in the energy harvesting system. Accordingly, the HEH changes into a conventional multi-frequency PEH (MPEH) with magnetic oscillator, as reported in reference [17]. When the piezoelectric layers are in the short-circuit condition, a conventional multi-frequency EMEH (MEMEH) with a magnetic oscillator can be developed. The magnetic oscillator and induction coil are the component units of the 1DOF linear EMEH (LEMEH). The combination of the LPEH and LEMEH is named LVEH. Its output power frequency response is compared with that of the HEH. 


\section{Results and Discussion}

To measure the optimal load resistance matched to the coil, the piezoelectric layers are short-circuit. There are two peak output powers for the configuration of two magnetic coupled oscillators. The first peak is induced by the magnetic oscillator, while the second one is induced by the piezoelectric oscillator. Figure 9 shows the first peak output power delivered to the load resistance $R_{2}$ for the MEMEH at resonant frequency. The maximum power is $1.2 \mathrm{~mW}$ at $22.8 \mathrm{~Hz}$ with the load resistance $370 \Omega$. The second peak output power delivered to the load resistance $R_{1}$ for the MPEH at resonant frequency is shown in Figure 10. The induction coil around the magnetic oscillator is open-circuit. With the increasing of $R_{1}$, the power reaches the maximum $2.57 \mathrm{~mW}$ at $57 \mathrm{k} \Omega$. The corresponding resonant frequency is $25.8 \mathrm{~Hz}$. By using the logarithmic decrement method, the measured mechanical damping ratios of piezoelectric beam and magnetic oscillator with magnetic interaction are 0.023 and 0.024 , respectively.

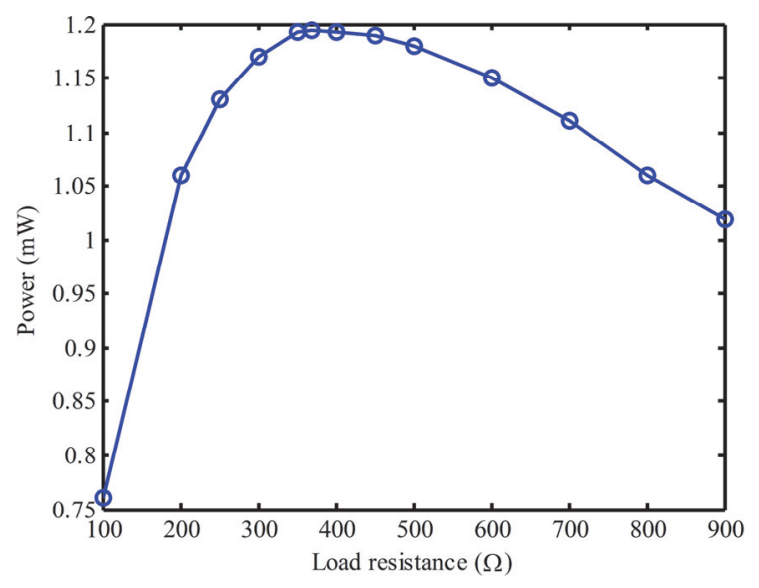

Figure 9. Output power with different load resistances for the MEMEH.

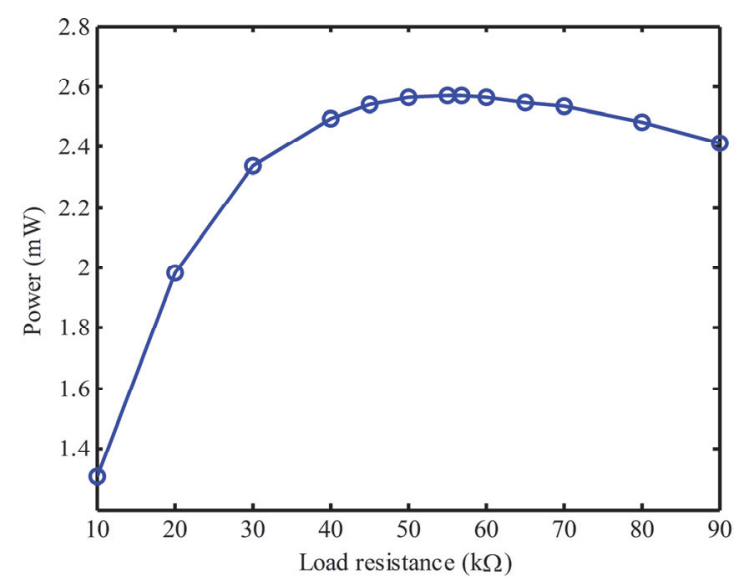

Figure 10. Output power with different load resistances for the MPEH.

Figure 11a,b shows the experimental and numerical comparisons of the frequency-response for the HEH, MPEH and MEMEH, respectively. The initial distance $d_{0}$ is still $52.5 \mathrm{~mm}$. The loads connected to the HEH are the optimal values determined from the MPEH and MEMEH. It can be seen in Figure 11a that each harvester has two peak output powers. For HEH, the measured peak output powers are $1.40 \mathrm{~mW}$ at $22.6 \mathrm{~Hz}$ and $2.82 \mathrm{~mW}$ at $25.6 \mathrm{~Hz}$. In view of the low-frequency applications, the first peak output power is the research focus. The first peak of the HEH increases by $16.7 \%$ and $833.3 \%$ compared with the first peaks of the MEMEH $(1.2 \mathrm{~mW})$ and the MPEH $(0.15 \mathrm{~mW})$, respectively. The frequency response of the HEH completely covers that of the MPEH and MEMEH. That is to say, the 
hybrid transduction mechanism is superior to the multi-frequency devices with single conversion mechanism in broadening the bandwidth and improving the generating performance. We can find that the simulation results of the HEH are in good agreement with the experimental results. The inaccuracies between theoretical and simulation results for the MPEH and MEMEH are significant. We think that these errors may be due to the damping change between the magnetic oscillator and the aluminum rod with varying vibration frequencies. Besides, the effective stiffness of the spring varies with the change of effective length during the oscillation, which may be another factor.
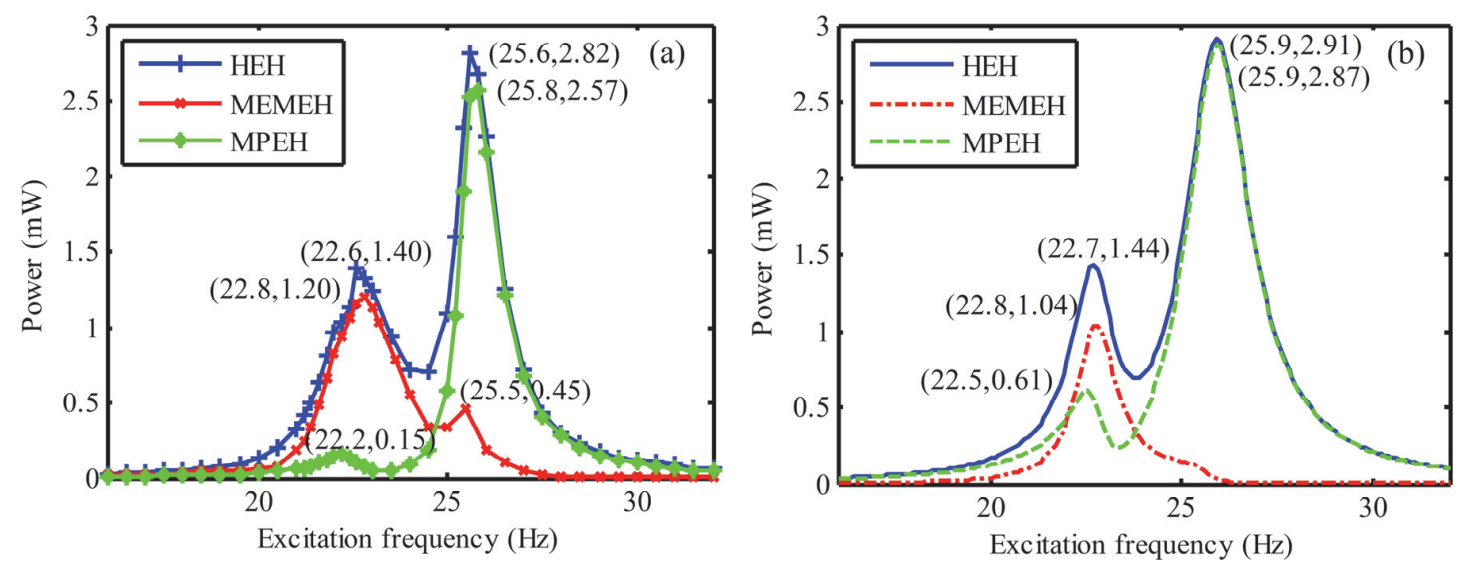

Figure 11. Frequency responses for the HEH, MEMEH, and MPEH: (a) experimental results; (b) numerical results.

Figure 12a,b illustrates the numerical and experimental comparisons of the output power for the HEH, LEMEH, LPEH and LVEHs, respectively. The connected load resistances keep still. Due to the repulsive magnetic force, the measured first resonance of the $\mathrm{HEH}(22.6 \mathrm{~Hz})$ is higher than that of the LVEHs $(21.6 \mathrm{~Hz})$ and the second one $(25.6 \mathrm{~Hz})$ is higher than that of the LVEHs $(25 \mathrm{~Hz})$. Moreover, the first peak power of the HEH $(1.4 \mathrm{~mW})$ is 2.36 times more than that of the LVEHs (the sum of $0.08 \mathrm{~mW}$ from LPEH and $0.49 \mathrm{~mW}$ from LEMEH) at the same excitation frequency. Meanwhile, it increases by $45.8 \%$ compared to that of the LVEHs, although the second peak power of the HEH decreases by $13.8 \%$. Obviously, the repulsive magnetic force can suppress the oscillation of the piezoelectric oscillator, but enhance the oscillation of the magnetic oscillator. The half-power bandwidth of the first peak power for the HEH is about $2.8 \mathrm{~Hz}$, which is wider than that of the LEMEH $(1.9 \mathrm{~Hz})$. It is clear that the generating performances of the LPEH and LEMEH are greatly improved by the magnetic coupling multi-frequency structure and hybrid transduction mechanism in the low-frequency range. Consequently, magnetic interaction is an effective approach to improve the performance of the micro energy harvester in the low-frequency range. The numerical results show good agreement with the experimental results. Note that the measured mechanical damping ratios of piezoelectric and magnetic oscillators without magnetic interaction are 0.025 and 0.02 , respectively. That is to say, magnetic interaction can add extra damping to the magnetic oscillator and cut down the damping of the piezoelectric oscillator. The simulation results for the LEMEH shows good agreement with the experimental results. Accordingly, the model of the electromagnetic coupling coefficient $\theta_{\mathrm{em}}$ is reasonable and valid. 

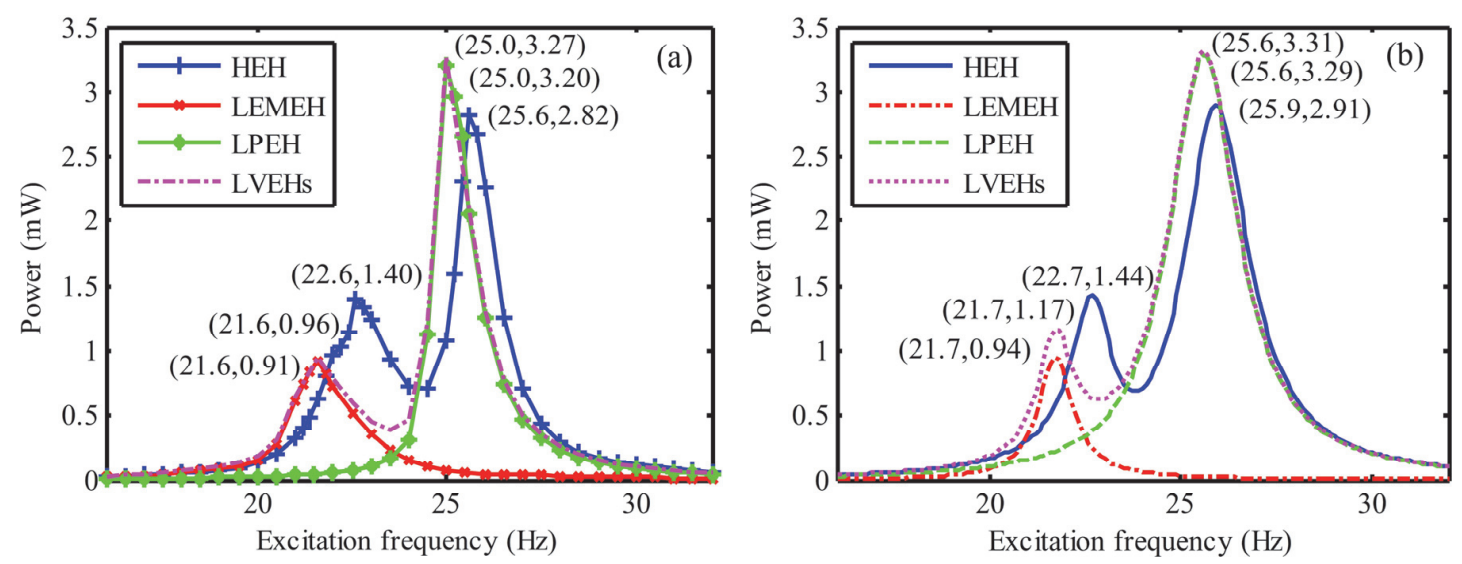

Figure 12. Frequency responses for the HEH, LEMEH, LPEH and LVEHs: (a) experimental results; (b) numerical results.

Figure 13 provides the investigation on the effects of the initial separation distance $d_{0}$ between two magnets on output power. The measurement was performed at three arbitrary different separation distances $42.5,47.0$, and $52.5 \mathrm{~mm}$. For the sake of contrastive analysis, the excitation acceleration was controlled at $2 \mathrm{~m} / \mathrm{s}^{2}$. It shows that the resonant frequencies shift to the right with the decreasing of the initial separation distance, which is consistent with the simulation result. It can be attributed to the increasing repulsive magnetic force and the magnetic stiffness. Consequently, the resonant frequencies can be tuned by adjusting the separation distance according to the ambient excitation frequency. Furthermore, the amplitude of the first peak output power is another issue which deserves concern. Contrary to the resonant frequency, the peak output power for the first resonance goes up continuously with the decreasing of the separation distance. In the future designing, we need to make a trade-off between the resonance and peak output power. With the decrease of $d_{0}$, the error between experimental and numerical results of the first peak power increases, because the mechanical damping ratios were kept constant in the theoretical model, while the actual values are variable. Moreover, the damping ratio of magnetic oscillator increases more significantly than that of the piezoelectric oscillator.
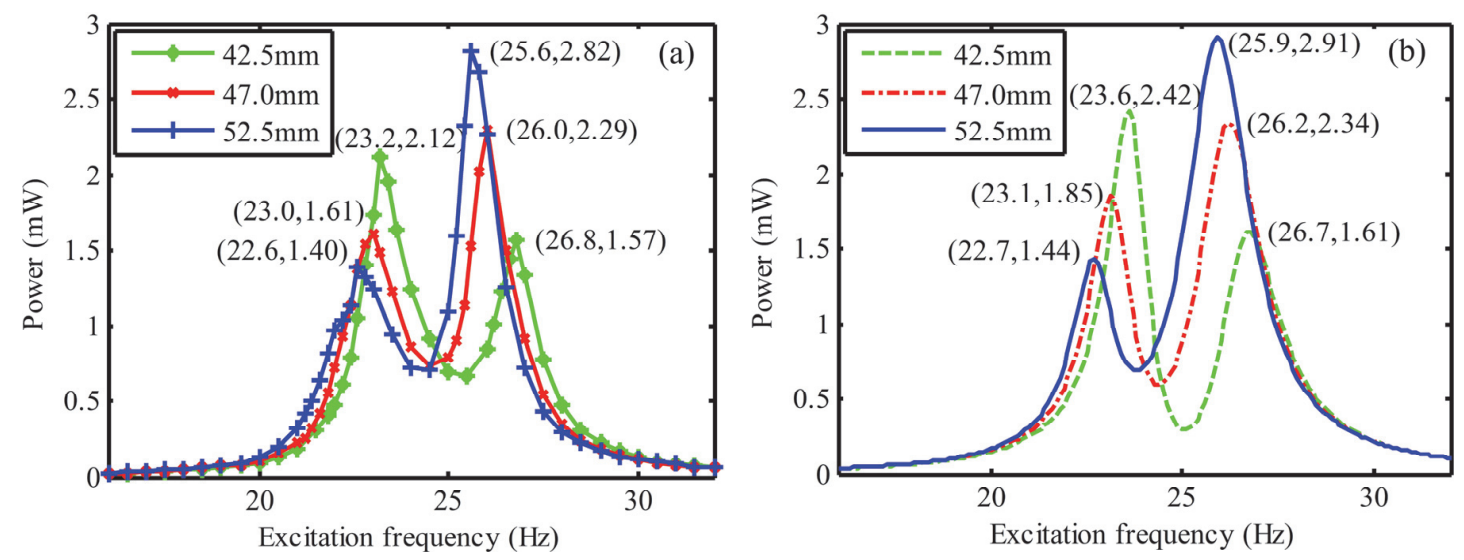

Figure 13. Frequency responses for the HEH with different separation distances $d_{0}$ : (a) experimental results; (b) numerical results.

Figure 14 shows the output power for the HEH with different directions of magnetic force. Two magnets will attract each other with the same magnetization direction, while they will repel each other in opposite direction. Due to the attractive force, the initial distance reduces to $47 \mathrm{~mm}$. As a result of the attractive force, the resonant frequencies shift to the left, while the first peak output power decreases sharply as compared to that of the HEH with repulsive force. On the contrary, the second peak output 
power is obviously enhanced. That is to say, the generating efficiency of piezoelectric element is much higher than that of the electromagnetic element with the attractive force. The theoretical value of the first peak power for the HEH is lower than the measured value. The reason is that attractive force induces more deformation of the piezoelectric beam. However, the mechanical damping of magnetic oscillator is also enhanced simultaneously. In this case, the HEH is more suitable for scavenging vibration energy in the high-frequency range. However, the attractive force is still a choice for energy harvesting at the lower excitation frequency. Similarly, the direction of magnetic force needs to be selected properly depending on the excitation frequency and electric power demands.
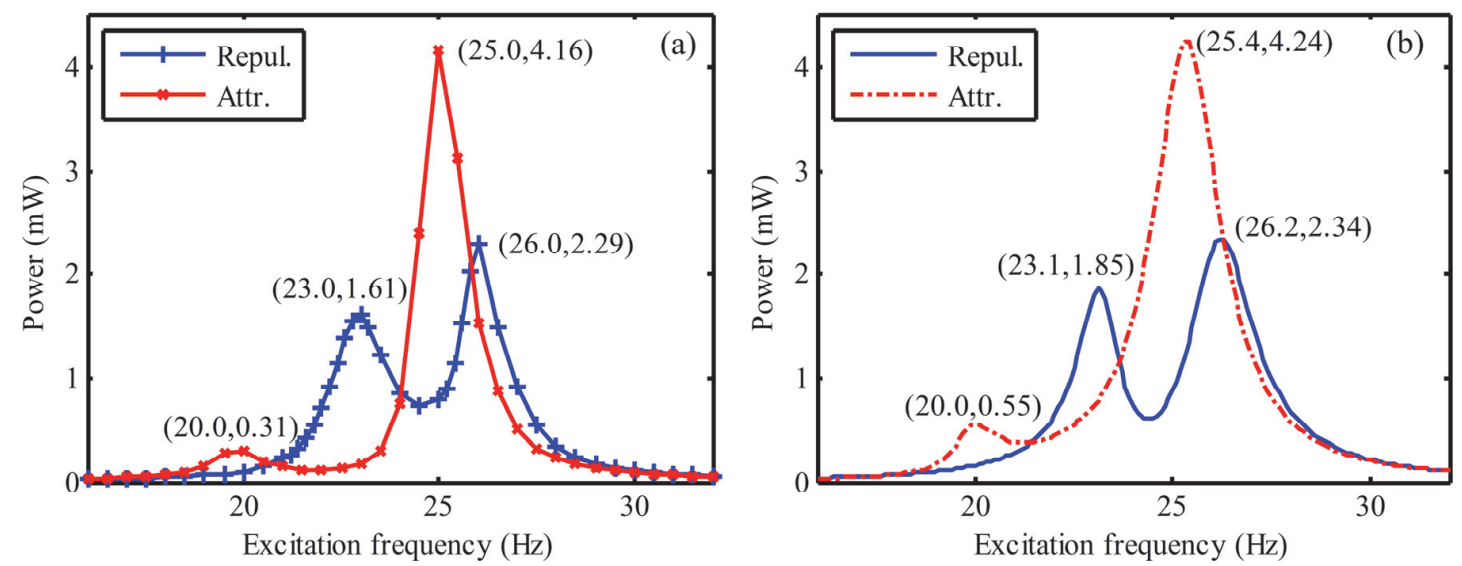

Figure 14. Frequency responses for the HEH with different directions of magnetic force: (a) experimental results; (b) numerical results.

\section{Conclusions}

In this paper, we proposed a novel tunable multi-frequency hybrid vibration energy harvester, by combining piezoelectric and electromagnetic transduction mechanisms. The electromechanical coupling model of the HEH was established and numerically simulated. The effects of magnetic fore, mass ratio, stiffness ratio, and mechanical damping ratios on the frequency response of the HEH were analyzed. A macro-scale prototype was fabricated and tested by experiments. It is demonstrated that magnetic interaction between piezoelectric and magnetic oscillators can tune the resonant frequencies of the HEH. It contributes to increasing energy harvesting efficiency of the micro energy harvesting devices in low-frequency range. The hybrid transduction mechanism is superior to the single mechanism in broadening the bandwidth and improving the output power. In addition, an initial separation distance between two magnets and the direction of magnetic force affect the frequency responses of the HEH significantly, which need to be carefully designed based on the excitation frequency and electric power demands. In a word, the proposed tunable multi-frequency hybrid energy harvesting mechanism is an effective approach to improve the performance of the micro-scale energy harvesting devices for the low-frequency environmental vibrations.

Acknowledgments: The work described in this paper is supported by "The Fundamental Research Funds for the Central Universities" (Grant No. HIT. NSRIF. 2014059 and No. HIT. KISTP. 201412), China.

Author Contributions: Zhenlong Xu established the theoretical model. All authors conceived and designed the experiments. Zhenlong $\mathrm{Xu}$ and Danpeng Chen performed the experiments. Zhenlong Xu, Xiaobiao Shan and Tao Xie analyzed the data. All authors contributed to the writing of the paper.

Conflicts of Interest: The authors declare no conflict of interest.

\section{References}

1. Roundy, S.; Wright, P.K. A piezoelectric vibration based generator for wireless electronics. Smart Mater. Struct. 2004, 13, 1131-1142. [CrossRef] 
2. Beeby, S.P.; Tudor, M.J.; White, N.M. Energy harvesting vibration sources for microsystems applications. Meas. Sci. Technol. 2006, 17, R175-R195. [CrossRef]

3. Williams, C.B.; Yates, R.B. Analysis of a micro-electric generator for microsystems. Sens. Actuators A Phys. 1996, 52, 8-11. [CrossRef]

4. Reilly, E.; Miller, L.; Fain, R.; Wright, P.K. A study of ambient vibrations for piezoelectric energy conversion. In Proceedings of the PowerMEMS, Washington, DC, USA, 1-4 December 2009; pp. 312-315.

5. Swee, L.K.; Neil, M.W.; Harris, N.R. A novel piezoelectric thick-film free-standing cantilever energy harvester. In Proceedings of the Eurosensors XXII, Dresden, Germany, 7-11 September 2008.

6. Elfrink, R.; Kamel, T.M.; Goedbloed, M.; Matova, S.; Hohlfeld, D.; van Andel, Y.; van Schaijk, R. Vibration energy harvesting with aluminum nitride-based piezoelectric devices. J. Micromech. Microeng. 2009, 19. [CrossRef]

7. Kim, M. Design, fabrication, and experimental demonstration of a piezoelectric cantilever for a low resonant frequency microelectromechanical system vibration energy harvester. J. Micro Nanolithogr. MEMS MOEMS 2012, 11. [CrossRef]

8. Alamin Dow, A.B.; Schneider, M.; Koo, D.; Al-Rubaye, H.A.; Bittner, A.; Schmid, U.; Kherani, N. Modeling the performance of a micromachined piezoelectric energy harvester. Microsyst. Technol. 2012, 18, 1035-1043. [CrossRef]

9. Beeby, S.P.; Tudor, M.J.; Torah, R.N.; Roberts, S.; O’Donnell, T.; Roy, S. Experimental comparison of macro and micro scale electromagnetic vibration powered generators. Microsyst. Technol. 2007, 13, 1647-1653. [CrossRef]

10. Shan, X.B.; Yuan, J.B.; Xie, T.; Chen, W.S. Design and experiment of multiple piezoelectric bimorphs for scavenging vibration energy. Int. J. Appl. Electromagn. Mech. 2010, 34, 265-275.

11. Sari, I.; Balkan, T.; Kulah, H. An electromagnetic micro power generator for wideband environmental vibrations. Sens. Actuators A Phys. 2008, 145-146, 405-413. [CrossRef]

12. Yang, Z.; Yang, J. Connected vibrating piezoelectric bimorph beams as a wide-band piezoelectric power harvester. J. Intell. Mater. Syst. Struct. 2009, 20, 569-574. [CrossRef]

13. Liu, H.; Tay, C.J.; Quan, C.; Kobayashi, T.; Lee, C. A scrape-through piezoelectric mems energy harvester with frequency broadband and up-conversion behaviors. Microsyst. Technol. 2011, 17, 1747-1754. [CrossRef]

14. Halim, M.A.; Park, J.Y. Theoretical modeling and analysis of mechanical impact driven and frequency up-converted piezoelectric energy harvester for low-frequency and wide-bandwidth operation. Sens. Actuators A Phys. 2014, 208, 56-65. [CrossRef]

15. Hajati, A.; Kim, S.-G. Ultra-wide bandwidth piezoelectric energy harvesting. Appl. Phys. Lett. $2011,99$. [CrossRef]

16. Cottone, F.; Basset, P.; Vocca, H.; Gammaitoni, L.; Bourouina, T. Bistable electromagnetic generator based on buckled beams for vibration energy harvesting. J. Intell. Mater. Syst. Struct. 2013, 25, 1484-1495. [CrossRef]

17. Tang, L.; Yang, Y. A nonlinear piezoelectric energy harvester with magnetic oscillator. Appl. Phys. Lett. 2012, 101. [CrossRef]

18. Mann, B.P.; Sims, N.D. Energy harvesting from the nonlinear oscillations of magnetic levitation. J. Sound Vib. 2009, 319, 515-530. [CrossRef]

19. Stanton, S.C.; Owens, B.A.M.; Mann, B.P. Harmonic balance analysis of the bistable piezoelectric inertial generator. J. Sound Vib. 2012, 331, 3617-3627. [CrossRef]

20. Stanton, S.C.; McGehee, C.C.; Mann, B.P. Reversible hysteresis for broadband magnetopiezoelastic energy harvesting. Appl. Phys. Lett. 2009, 95. [CrossRef]

21. Mahmoudi, S.; Kacem, N.; Bouhaddi, N. Enhancement of the performance of a hybrid nonlinear vibration energy harvester based on piezoelectric and electromagnetic transductions. Smart Mater. Struct. 2014, 23. [CrossRef]

22. Shan, X.; Xu, Z.; Song, R.; Xie, T. A new mathematical model for a piezoelectric-electromagnetic hybrid energy harvester. Ferroelectrics 2013, 450, 57-65. [CrossRef]

23. Challa, V.R.; Prasad, M.G.; Shi, Y.; Fisher, F.T. A vibration energy harvesting device with bidirectional resonance frequency tunability. Smart Mater. Struct. 2008, 17. [CrossRef]

24. Zhu, D.; Roberts, S.; Tudor, M.J.; Beeby, S.P. Design and experimental characterization of a tunable vibration-based electromagnetic micro-generator. Sens. Actuators A Phys. 2010, 158, 284-293. [CrossRef] 
25. Peters, C.; Maurath, D.; Schock, W.; Mezger, F.; Manoli, Y. A closed-loop wide-range tunable mechanical resonator for energy harvesting systems. J. Micromech. Microeng. 2009, 19. [CrossRef]

26. Chew, Z.; Li, L. Design and characterisation of a piezoelectric scavenging device with multiple resonant frequencies. Sens. Actuators A Phys. 2010, 162, 82-92. [CrossRef]

27. Ferrari, M.; Ferrari, V.; Guizzetti, M.; Marioli, D.; Taroni, A. piezoelectric multifrequency energy converter for power harvesting in autonomous microsystems. Sens. Actuators A Phys. 2008, 142, 329-335. [CrossRef]

28. Yang, B.; Lee, C.; Xiang, W.; Xie, J.; He, J.H.; Kotlanka, R.K.; Low, S.P.; Feng, H. Electromagnetic energy harvesting from vibrations of multiple frequencies. J. Micromech. Microeng. 2009, 19. [CrossRef]

29. Yang, B.; Liu, J.; Tang, G.; Luo, J.; Yang, C.; Li, Y. A generator with nonlinear spring oscillator to provide vibrations of multi-frequency. Appl. Phys. Lett. 2011, 99. [CrossRef]

30. Liu, H.; Soon, B.W.; Wang, N.; Tay, C.J.; Quan, C.; Lee, C. Feasibility study of a 3D vibration-driven electromagnetic mems energy harvester with multiple vibration modes. J. Micromech. Microeng. $2012,22$. [CrossRef]

31. Tadesse, Y.; Zhang, S.; Priya, S. Multimodal energy harvesting system: Piezoelectric and electromagnetic. J. Intell. Mater. Syst. Struct. 2009, 20, 625-632. [CrossRef]

32. Wang, H.-Y.; Tang, L.-H.; Guo, Y.; Shan, X.-B.; Xie, T. A 2DOF hybrid energy harvester based on combined piezoelectric and electromagnetic conversion mechanisms. J. Zhejiang Univ. Sci. A 2014, 15, 711-722. [CrossRef]

33. Shan, X.-B.; Guan, S.-W.; Liu, Z.-S.; Xu, Z.-L.; Xie, T. A new energy harvester using a piezoelectric and suspension electromagnetic mechanism. J. Zhejiang Univ. Sci. A 2013, 14, 890-897. [CrossRef]

34. Spreemann, D.; Hoffmann, D.; Folkmer, B.; Manoli, Y. Numerical optimization approach for resonant electromagnetic vibration transducer designed for random vibration. J. Micromech. Microeng. 2008, 18. [CrossRef]

35. Yung, K.W.; Landecker, P.B.; Villani, D.D. An analytic solution for the force between two magnetic dipoles. Magn. Electr. Sep. 1998, 9, 39-52. [CrossRef]

36. Sneller, A.J.; Mann, B.P. On the nonlinear electromagnetic coupling between a coil and an oscillating magnet. J. Phys. D Appl. Phys. 2010, 43. [CrossRef]

37. Priya, S.; Inman, D.J. Energy Harvesting Technologies; Springer: New York, NY, USA, 2009; p. 132.

38. DuToit, N.E.; Wardle, B.L. Experimental verification of models for microfabricated piezoelectric vibration energy harvesters. AIAA J. 2007, 45, 1126-1137. [CrossRef]

39. Sodano, H.A.; Park, G.; Inman, D.J. Estimation of electric charge output for piezoelectric energy harvesting. Strain 2004, 40, 49-58. [CrossRef]

40. Roundy, S.; Wright, P.K.; Rabaey, J. A study of low level vibrations as a power source for wireless sensor nodes. Comput. Commun. 2003, 26, 1131-1144. [CrossRef]

41. Inman, D.J.; Singh, R.C. Engineering Vibration, 3rd ed.; Pearson Education: Hackensack, NJ, USA, 2008; pp. $43-48$.

42. Stephen, N.G. On energy harvesting from ambient vibration. J. Sound Vib. 2006, 293, 409-425. [CrossRef]

43. Erturk, A.; Inman, D.J. Piezoelectric Energy Harvesting; John Wiley \& Sons: West Sussex, UK, 2011; pp. $373-374$.

(C) 2015 by the authors; licensee MDPI, Basel, Switzerland. This article is an open access article distributed under the terms and conditions of the Creative Commons by Attribution (CC-BY) license (http:/ / creativecommons.org/licenses/by/4.0/). 